\section{Scleroderma models: skin in the game}

\section{By Michael J. Haas, Senior Writer}

Systemic scleroderma involves fibrosis of the skin and internal organs, but its etiology is poorly understood and it has no known genetic causes-factors that have hampered the development of models and, in turn, therapies.

Now, a North American team has shown that mice harboring a mutant form of the glycoprotein fibrillin 1 (Fbn 1 ) recapitulated skin fibrosis and other symptoms seen in patients with systemic scleroderma. ${ }^{1}$ Although the team showed the therapeutic effect of targeting different proteins whose expression was altered by the mutant glycoprotein, future studies will have to zero in on the best target for treating scleroderma in patients.

FBN1, a glycoprotein secreted by fibroblasts into the extracellular matrix, is an essential component of the microfibrils found in many types of connective tissue. FBN1 also interacts with integrins expressed on other cell types,

"These mouse models
could help us study the
biology behind scleroderma
and identify therapeutic
strategies for preventing or
reversing skin fibrosis."
-Luke Evnin,
Scleroderma Research Foundation

anti-nuclear and anti-topoisomerase I (Top1) antibodies in circulation seen in patients with systemic scleroderma.

Plasmacytoid DCs isolated from the dermis of the mutant mice and fibroblasts from patients with systemic scleroderma had high surface levels of integrin $\beta_{1}$ (CD29) and activated integrin $\beta_{3}$ (GPIIIa; CD61).

In the Fbn 1-mutant mice, a mouse $\mathrm{Cd} 29$-activating antibody-which mimicked Fbn1's interactions with Cd29-decreased skin fibrosis and levels of circulating anti-nuclear and anti-Top1 antibodies compared with an inactive murine control antibody.

Knockout of $C d 61$ or a pan-specific, anti-Tgf $\beta$ antibody also decreased skin fibrosis in the mice compared with normal Cd61 expression or a control antibody.

In the patient fibroblasts, a CD29-activating antibody, an anti-CD61 antibody or a small molecule against type I TGF $\beta$ receptor decreased collagen expression compared with inactive control antibodies.

Additionally, patient fibroblasts and DCs from the dermis of the mouse models had elevated TGF $\beta$-dependent MAP kinase 3 (MAPK3; ERK-1) and/or MAPK1 (ERK-2) signaling. In the fibroblasts and models, a small molecule MEK inhibitor decreased collagen expression and skin fibrosis, respectively, compared with vehicle.

Collectively, the findings suggest that stiff skin syndrome and systemic scleroderma involve similar pathological mechanisms (see Figure 1, "Restoring dermal integrity") and thus could potentially be treated with the same therapeutic strategies, the team wrote in its report in Nature.

There are no drugs approved to treat stiff skin syndrome or the underlying causes of such as dermal-infiltrating dendritic cells (DCs), to regulate adhesion.

FBN1 mutations can cause a number of different conditions and diseases that affect connective tissue, most notably Marfan syndrome. ${ }^{2}$

In 2006, a team at The Johns Hopkins University School of Medicine led by Harry Dietz showed that some FBN1 mutations could upregulate signaling by transforming growth factor- $\beta$ (TGFB; TGF $\beta$ ) - a family of cytokines that is involved in the proliferation and differentiation of most cells-to cause aortic aneurysms that occur in some patients with Marfan syndrome. ${ }^{3}$

Dietz's team also identified a few patients harboring FBN1 mutations who exhibited symptoms of both Marfan syndrome and stiff skin syndrome, a rare inherited form of skin fibrosis with only about 40 cases reported in the literature. Subsequently, another Dietz-led team determined that stiff skin syndrome was caused by mutations in the integrin-binding domain of FBN1 and involved upregulation of TGF $\beta$ signaling. ${ }^{4}$

Now, his newest team has hypothesized that mice with loss-offunction mutations in the integrin-binding domain of $F b n 1$ might provide insights into the pathobiology of another fibrotic disease associated with increased TGF $\beta$ signaling - scleroderma. $^{5}$

Indeed, the team found that the Fbn 1-mutant mice exhibited skin fibrosis, high levels of collagen in the skin and the high levels of systemic scleroderma. Therapies for the latter primarily involve topical or systemic immunosuppressive drugs to ameliorate symptoms.

Dietz is a professor of pediatrics, medicine, and molecular biology and genetics at the Johns Hopkins University School of Medicine and a professor of medicine and genetics at the school's McKusick-Nathans Institute for Genetic Medicine. He is also director of the school's William S. Smilow Center for Marfan Syndrome Research and an investigator at the Howard Hughes Medical Institute. His team included a researcher from McGill University.

Although stiff skin syndrome and scleroderma have differing or unknown causes, "Dietz has shown that, once initiated, the diseases appear to have similar biological processes that converge on the same pathways," Luke Evnin, chairman of the Scleroderma Research Foundation, told SciBX. "These mouse models could help us study the biology behind scleroderma and identify therapeutic strategies for preventing or reversing skin fibrosis."

Evnin, who is a managing director at life sciences VC MPM Capital, said that the Scleroderma Research Foundation recruited Dietz about six years ago to work on scleroderma and funded the research in the Nature study.

"Among the obstacles to understanding scleroderma are the heterogeneity of the disease-systemic sclerosis that affects multiple 
InterMune Inc. (NASDAQ:ITMN), Ildong Pharmaceutical Co. Ltd. and Shionogi \& Co. Ltd. (Tokyo:4507) market Esbriet pirfenidone, a small molecule inhibitor of profibrotic cytokines such as transforming growth factor- $\beta$ (TGFB; TGF $\beta$ ) and proinflammatory cytokines such as tumor necrosis factor- $\alpha$ (TNF- $\alpha$ ), to treat idiopathic pulmonary fibrosis (IPF); Eli Lilly and Co. (NYSE:LLY) has LY2382770, a neutralizing mAb against TGF 1 1 (TGFB1) in Phase II for diabetic nephropathy; Digna Biotech S.L. has disitertide (P144), a topical formulation of peptide 144 TGFB1 inhibitor, in Phase II for systemic sclerosis and Phase I for actinic keratosis (AK); Isarna Therapeutics GmbH has trabedersen (AP 12009), a TGF $\beta 2$ (TGFB2) antisense oligonucleotide, in Phase II for pancreatic cancer and melanoma and Phase I/II for colorectal cancer; Yuhan Corp. has YH14618, a TGFB1 antagonist, in Phase I/II for degenerative disc disease.

Exelixis Inc. (NASDAQ:EXEL), Roche (SIX:ROG; OTCQX:RHHBY) and Genentech Inc. have the MEK inhibitor cobimetinib (GDC-0973; RG7421; XL518) in Phase III for melanoma; Array

BioPharma Inc. (NASDAQ:ARRY) and AstraZeneca plc (LSE:AZN; NYSE:AZN) have selumetinib (ARRY-886; AZD6244), a small molecule MEK inhibitor, in Phase III for non-small cell lung cancer (NSCLC) and Phase II for multiple other cancers; Array and Novartis AG (NYSE:NVS; SIX:NOVN) have MEK162 (ARRY-162), an oral MEK inhibitor, in Phase III for melanoma and ovarian cancer, Phase II for solid tumors and hypertrophic cardiomyopathy $(\mathrm{HCM})$ in patients with Noonan syndome, Phase I/II for bilary tract cancer and Phase I for colorectal cancer; Bayer AG (Xetra:BAYN) and AstraZeneca have refametinib (BAY 86-9766; RDEA119), a selective MEK inhibitor, in Phase II for hepatocellular carcinoma (HCC) and Phase I/II for pancreatic cancer; UCB Group (Euronext:UCB) and Wilex AG (Xetra:WL6) have MK-554 (WZ-554), an orally available small molecule MEK inhibitor, in Phase I/II for solid tumors.

Figure 1. Restoring dermal integrity. A study from Gerber et al. has elucidated potential mechanisms by which mutations in the integrin-binding domain of fibrillin 1 (Fbn1) produce symptoms of stiff skin syndrome and systemic scleroderma. The findings suggest that the biological processes of the two diseases converge on shared signaling pathways that offer common intervention points.

In mouse dermis, mutant Fbn1 induces two distinct pathways that produce activated transforming growth factor- $\beta$ (Tgfb; Tgf $\beta$ ) and activated (*) integrin $\beta_{3}$ (Gpllla; Cd61), respectively -two components required for collagen expression and consequent skin fibrosis.

Mutant Fbn1 [a(1)] secreted by fibroblasts into the extracellular matrix binds $\operatorname{Tgf} \beta[\mathbf{b}(1)]$ normally but forms a large number of abnormal microfibrillar aggregates, resulting in high levels of latent

organs-and the variability of its clinical course. The Nature study helps reduce this complexity by focusing on a genetic model involving only pathological fibrosis of the skin," said Gary Nabel, CSO of Sanofi.

Thus, this high-fidelity model of skin scleroderma could be used to screen for new compounds to treat the disease or possibly repurpose those in development for other diseases, he said.

Sanofi's SAR100842, an antagonist of lysophosphatidic acid receptor 1 (LPAR1; EDG2; LPA1) and LPAR3 (EDG7; LPA3), is in Phase II testing to treat scleroderma. LPA antagonists are thought to modify TGF $\beta$ activity indirectly, Nabel said.

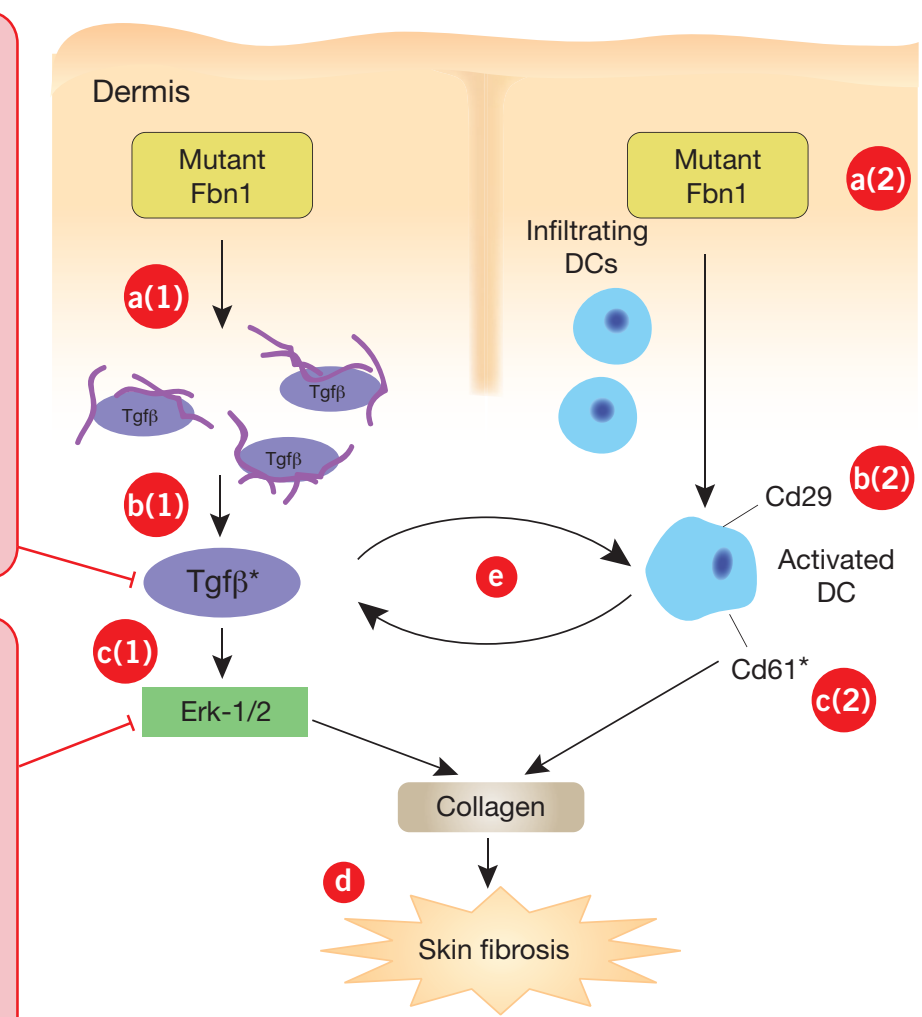

$\operatorname{Tgf} \beta$ that become activated and upregulate $\operatorname{Tgf} \beta^{\star}$-dependent MAP kinase 3 (Mapk3; Erk-1) and/or Mapk1 (Erk-2) signaling [c(1)].

Also in the dermis, Fbn1 cannot interact normally with infiltrating plasmacytoid dendritic cells (DCs) [a(2)], causing them to upregulate surface expression of integrin $\beta_{1}(\mathrm{Cd} 29)[\mathbf{b}(2)]$ and $\mathrm{Cd} 61^{\star}[\mathrm{c}(2)]$.

Together, increased Tgf $\beta$-dependent Erk-1/2 signaling and high Cd61* levels lead to excessive collagen production and consequent skin fibrosis [d].

The two pathways also may be involved in a feedback loop [e] by which $\operatorname{Tgf} \beta{ }^{*}$ induces infiltrating DCs to produce additional Tgf $\beta$.

Fibroblasts from patients with systemic scleroderma also express high levels of TGF $\beta[\mathbf{b}(\mathbf{1})]$, have high surface levels of CD29 [b(2)] and CD61* [c(2)] and upregulate ERK-1/2 signaling [c(1)] to induce collagen production [d].

Inhibition of $\operatorname{Tgf} \beta$, activation of $\mathrm{Cd} 29$, inhibition or deletion of Cd61 or inhibition of Erk1/2 in the mouse models and patient fibroblasts decreased skin fibrosis and collagen production, respectively, compared with controls.

\section{Going more than skin deep}

Further work is needed before deciding which therapeutic strategy-targeting CD29, CD61 or TGF $\beta$ - has the best chance in scleroderma.

"A large body of evidence points to a role of TGF $\beta$ in scleroderma, and Dietz's team's work further supports this," said Thomas Hultsch, senior medical director of translational medicine at Sanofi's Genzyme Corp. unit. "Unraveling the mechanisms that control TGF $\beta$ activation in the microenvironment of the dermis will be central for further development" of the team's findings. 
Added Nabel, "Our reading is that Dietz suggests the integrin pathways may allow modulation of TGF $\beta$ in a more controlled way, spatially and temporally, than targeting the cytokine directly. However, this claim remains preliminary at the present time."

Evnin agreed and said that targeting integrins $\beta_{1}$ or $\beta_{3}$ would probably be a better strategy than inhibiting TGF $\beta$ "because the cytokine is involved in many biological processes and thus is not a good target for a chronic disease like scleroderma."

He added, "An obvious next step would be to compare the relative efficacy and safety of the integrin $\beta_{3}$-blocking and integrin $\beta_{1}$-activating approaches in the Fbn1-mutant mouse models."

Hultsch said that it also would be interesting to dissect the role of the specific TGF $\beta$ isoforms TGF $\beta 1$ (TGFB1), TGF $\beta 2$ (TGFB2) and TGF $\beta 3$ (TGFB3) in skin fibrosis using isoform-specific antibodies, inhibitors or knockout models.

Evnin said that one drawback of the Fbn1-mutant models is that they do not exhibit the potentially life-threatening internal organ fibrosis seen in patients with systemic scleroderma. "That does leave open to question whether targeting integrins or TGF $\beta$ would also prevent or reverse that fibrosis. While this question can't be answered in mice, our hope is that the therapeutic effect would indeed be the same," he said.

According to Evnin, The Johns Hopkins University has filed a patent application covering the findings reported in Nature.

Dietz did not respond to queries about his team's follow-on studies in the Fbn 1 models.

This week, Biogen Idec Inc. and the BioFocus subsidiary of Galapagos N.V. announced a three-year collaboration to identify and validate new targets in scleroderma. Under the terms of the deal, BioFocus will use its technology platform and human skin models to deliver new assays and previously unknown, validated targets to Biogen Idec. Galapagos said that the deal could net BioFocus up to $\$ 31$ million. Other terms were not disclosed.

Haas, M.J. SciBX 6(44); doi:10.1038/scibx.2013.1248

Published online Nov. 14, 2013

\section{REFERENCES}

1. Gerber, E.E. et al. Nature; published online Oct. 9, 2013; doi:10.1038/nature12614

Contact: Harry C. Dietz, The Johns Hopkins University School of Medicine, Baltimore, Md. e-mail: hdietz@jhmi.edu

2. Dietz, H.C. et al. Nature 352, 337-339 (1991)

3. Habashi, J.P. et al. Science 312, 117-121 (2006)

4. Loeys, B.L. et al. Sci. Transl. Med. 2, 23ra20 (2010)

5. Varga, J. \& Pasche, B. Nat. Rev. Rheumatol. 5, 200-206 (2009)

\section{COMPANIES AND INSTITUTIONS MENTIONED}

Biogen Idec Inc. (NASDAQ:BIIB), Weston, Mass.

The Johns Hopkins University, Baltimore, Md.

The Johns Hopkins University School of Medicine, Baltimore, Md.

Galapagos N.V. (Euronext:GLPG; Pink:GLPYY), Mechelen, Belgium Genzyme Corp., Cambridge, Mass.

Howard Hughes Medical Institute, Chevy Chase, Md.

McGill University, Montreal, Quebec, Canada

MPM Capital, South San Francisco, Calif.

Sanofi (Euronext:SAN; NYSE:SNY), Paris, France

Scleroderma Research Foundation, San Francisco, Calif. 